\title{
Horizontal Rotary Cutting Technique for Skin Injury Avoidance in Superficial Benign Breast Masses
}

\author{
Ren-Feng Song ${ }^{1} *$ \\ Yu-Xia Jiang ${ }^{2, *}$ \\ Jian-Chun Cui ${ }^{3}$ \\ Xue-Wei Yu' \\ Yang $\mathrm{Gao}^{3}$ \\ $\mathrm{Li} \mathrm{Li}^{3}$ \\ Xiao-Hua $\mathrm{Pei}^{4}$
}

'Department of General Surgery, The First People's Hospital of Huangzhong District, Xining, 811600, People's Republic of China; ${ }^{2}$ Department of Thyroid and Breast Surgery, The Chinese Medicine Hospital of Shexian, Handan, 056400, People's Republic of China; ${ }^{3}$ Department of Thyroid and Breast Surgery, People's Hospital of China Medical University (Liaoning Provincial People's Hospital), Shenyang, II00I5, People's Republic of China; ${ }^{4}$ Department of General Surgery, Xiamen Hospital of Beijing University of Traditional Chinese Medicine, Xiamen, 36I00I, People's Republic of China

*These authors contributed equally to this work

Correspondence: Jian-Chun Cui Department of Thyroid and Breast Surgery, People's Hospital of China Medical University (Liaoning Provincial People's Hospital), No. 33 of Wenyi Street, Shenhe District, Shenyang,

I I00 I5, People's Republic of China

$\mathrm{Tel} / \mathrm{Fax}+86-2424016014$

Email cui_jc8e@I63.com

Xiao-Hua Pei

Department of Surgery, Xiamen Hospital of Beijing University of Traditional

Chinese Medicine, No. 1739 of Xianyue

Street, Huli District, Xiamen, 36I00I,

People's Republic of China

Tel/Fax +86-5925579686

Email pxhertt@I63.com
Objective: This study aims to investigate the feasibility of the horizontal rotary-cut technique in the removal of superficial benign breast tumors with a $\leq 1.0 \mathrm{~cm}$ distance between the upper margin of the tumor and the skin.

Patients and Methods: A total of 69 patients with superficial benign breast tumors received horizontal rotary-cut surgery between July 2018 and June 2019 (horizontal group). The rotary cutter groove was in the true lateral position of the tumor and the ultrasonic probe was vertical to the rotary cutter groove. The patients were compared with 33 patients who underwent the traditional vertical rotary-cut surgery between July 2017 and June 2018 (traditional group) regarding the aspects of operation time, intraoperative bleeding volume, postoperative skin ecchymosis, skin damage, and tumor residue. The rotary cutter groove was directly below the tumor and the ultrasonic probe was parallel to the rotary cutter groove in the traditional vertical rotary-cut surgery.

Results: The operation time in the horizontal group was significantly shorter than in the traditional group $(7.7 \pm 1.1$ minutes vs $9.5 \pm 1.3$ minutes, with $t=-7.458$ and $\mathrm{p}=0.000)$ and there was significantly less skin damage in the horizontal group than in the traditional group ( 0 cases vs 3 cases, with $\mathrm{p}=0.032$ ). The differences in intraoperative bleeding and postoperative skin ecchymosis between the two groups were not statistically significant $(6.0 \pm 1.3 \mathrm{~mL}$ vs $6.5 \pm 1.5 \mathrm{~mL}$, with $t=-1.853$ and $\mathrm{p}=0.067 ; 4$ cases vs 2 cases, with $\chi^{2}=0.003$ and $\left.\mathrm{p}=0.958\right)$. Ninety-seven patients attended follow-ups for $6-30(16.5 \pm 4.5)$ months. No residues or recurrences were observed under ultrasound reviews in either group.

Conclusion: In superficial benign breast tumor removal, the horizontal rotary-cut breast technique can help avoid skin injury, shorten the operation time, and reduce tumor residue more effectively compared with the traditional vertical rotary-cut technique. It has certain popularization and application values.

Keywords: three-dimensional stereotaxis, horizontal, rotary-cut/rotary cutting, superficial, breast tumors/masses

\section{Introduction}

In 1998, a vacuum-assisted breast biopsy system (breast rotary-cut technique) was applied in the treatment of breast tumors by Zannis et al, ${ }^{1}$ and in 2004, the Mammotome was approved for the treatment of benign breast diseases by the United States Food and Drug Administration (USFDA). ${ }^{2}$ With the mass above the cutter groove and the ultrasonic probe, breast mass, and rotary cutter groove 


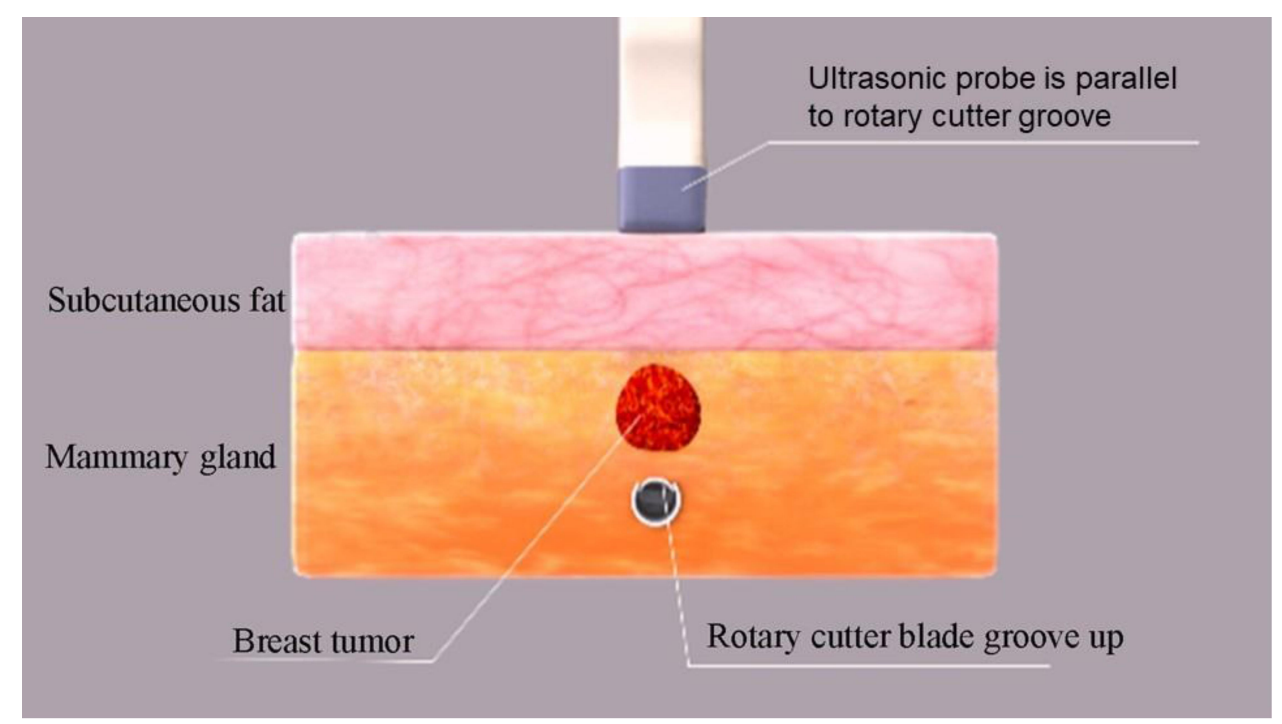

Figure I Schematic illustration of traditional vertical rotary cutting. The rotary cutter slot is in the middle and lower part of the breast mass and the ultrasound probe is parallel to the rotary cutter slot.

plane perpendicular to the chest wall, this technique is considered a classical operational approach (Figure 1). ${ }^{3}$ Although this type of vertical rotary cutting is intuitive and easy for clinicians to master quickly, there is still a risk ${ }^{4}$ of skin damage. The traditional technology has been improved in clinical practice and the horizontal rotary-cut technique applied in rotary-cut surgery of superficial benign breast tumors with a $\leq 1.0 \mathrm{~cm}$ distance between the upper edge of the tumor and the skin (Figure 2). The method has obtained a good outcome. It is reported as follows.

\section{Clinical Data and Methods}

\section{General Information}

A total of 102 female patients (58 in the People's Hospital of China Medical University, 16 in the First People's Hospital of Huangzhong, Qinghai, 18 in Handan Hospital of Traditional Chinese Medicine, Hebei, and 10 in Xiamen Hospital of Beijing University of Traditional Chinese Medicine) from July 2017 to June 2019 were included in this study. Inclusion criteria: (1) ultrasonography showed a single tumor; (2) the upper margin of the tumor was located $0.4-1.0 \mathrm{~cm}$ from the skin and the tumor had

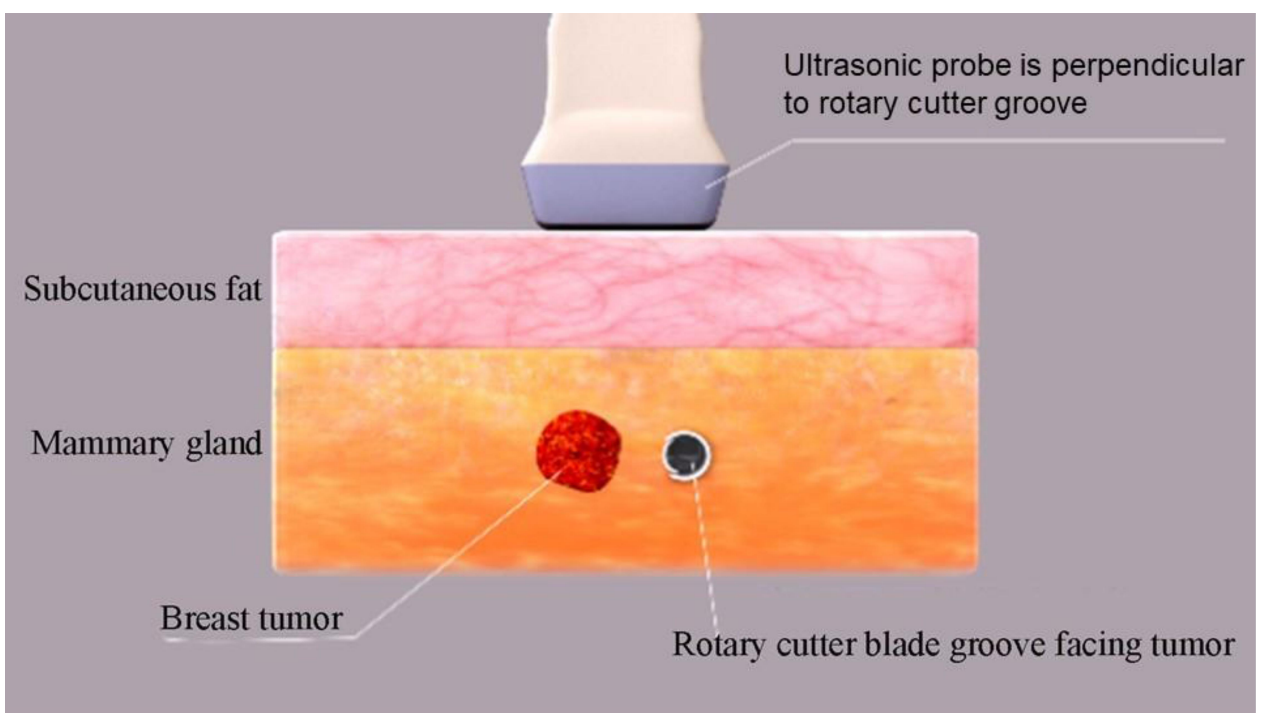

Figure 2 Schematic illustration of horizontal rotary cutting. The rotary cutter slot is directly lateral to the level of the breast mass and the ultrasound probe is perpendicular to the rotary cutter slot. 
a diameter of $0.5-1.9 \mathrm{~cm}$; (3) the tumor ultrasound either showed BI-RADS II-III and the patient was anxious and refused observation, or it showed BI-RADS class IVA. Exclusion criteria: (1) patients were in their menstrual period or had bleeding tendencies; (2) patients had cardiopulmonary disease and were unable to tolerate the surgery; (3) the tumor was located within $1.0 \mathrm{~cm}$ of the nipple and its surroundings.

The study was conducted in accordance with the Declaration of Helsinki (as revised in 2013) and was approved by the ethics committee of the People's Hospital of China Medical University (Liaoning Provincial People's Hospital). Informed consent was taken from all patients.

\section{Patients and Methods}

\section{Instruments}

ENCOR host, made by Bard, USA (Model: DR ENCOR; Approval No.: GSYJX [Jin] Zi 2013 3541849\#); rotary cutter bit (model: ECP017G; Approval No.: GXZJ 20173156309); 7G cutter bit with a $19 \mathrm{~mm}$ groove; color ultrasound (Model: LOGIQP3, GE) with a 7.5-10 MH ultrasonic probe frequency. The body surface positioning scale membrane (mammary gland scale membrane, patent no.: ZL 201020172673.2) for breast tumors was used for preoperative positioning and intraoperative reduction, as well as for postoperative review comparisons before and after the operation.

\section{Surgical methods}

The nature, quantity, size, and location of the tumors shown in the ultrasound report were reviewed before the operation in order to clarify and define the nature, quantity, size, and location of the tumor requiring removal. Next, the surface of the skin was marked in the location of the tumor and the mammary gland scale membrane was fastened. The surgical scope for the tumor was extended in preparation for the intraoperative reduction. Depending on the diameter of the tumor, either a full-cutter groove or half-cutter groove was selected for the surgery.

The patients were placed in a supine position under local anesthesia and hemodynamic monitoring. Lidocaine and epinephrine injections ( $\leq 80 \mathrm{~mL}$, each) were used around the tumor and in the puncture needle passage, subcutaneous adipose layer, and posterior space of the mammary gland for infiltration anesthesia. ${ }^{3}$ A small, $0.3-0.5 \mathrm{~cm}$ incision was made as a puncture mouth either along the areola margin or in the lateral mammary gland, according to the breast tumor position. The other incisions were made in the anterior axillary line, except for an incision in the areola margin in the case of a medial semimammary tumor near the sternum.

Horizontal group: The rotary cutter bit was inserted into the puncture mouth. A three-step approach was performed under ultrasound, in the order of coronal, sagittal, and horizontal position, to locate the relative position of the breast tumor and rotary cutter groove, thus ensuring that the cutter groove was completely located in a horizontal, true lateral position of the breast tumor. Horizontal cutting was then completed by pressing the "Cutting" key (Figure 3A-C). During the cutting procedure, the ultrasonic probe was perpendicular to the cutter groove. The angle and position of the cutter groove were adjusted at any time during the procedure in accordance with the cross-section of the tumor in order to remove $\mathrm{it}^{5,6}$ quickly and completely.

Traditional group: The rotary cutter bit was inserted into the puncture mouth, and the cutter groove was inserted directly below the tumor under ultrasound (Figure 1) during sector cutting.

The ultrasound probe cross-cutting method was introduced to determine whether breast tumor residue was present. If there was no active bleeding after removing the tumor, the puncture mouth was folded with a dressing patch and filled locally with dry gauze. Cotton pads were then applied around the chest. A self-adhesive elastic bandage was used for pressure bandaging and the affected upper arm was immobilized using a sling. The dressings were changed three days after surgery. If there were no obvious hematomas present, a sports bra was used for five to seven days for breast fixation. If hematomas were present, paracentesis was performed under ultrasound (breast ultrasounds were reviewed six months after the operation), and detected breast masses were once again marked on the body surface with a marker pen. A mammary gland scale membrane was applied for scope extension and compared with the preoperative mammary gland scale membrane to clarify whether there was any residue or recurrence in the tumor resection site.

\section{Observational Indicators}

Observational indicators consisted of surgical resection time (including the time of ultrasonic location and rotarycut operation and excluding the time of anesthesia and compression hemostasis), local residue or recurrence six 
A

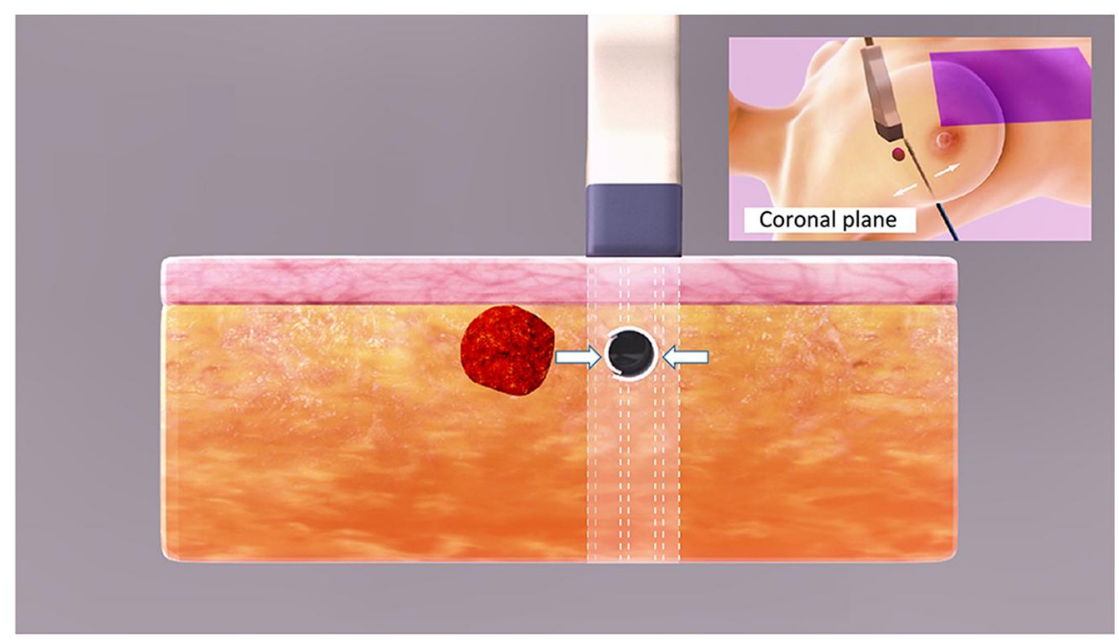

B

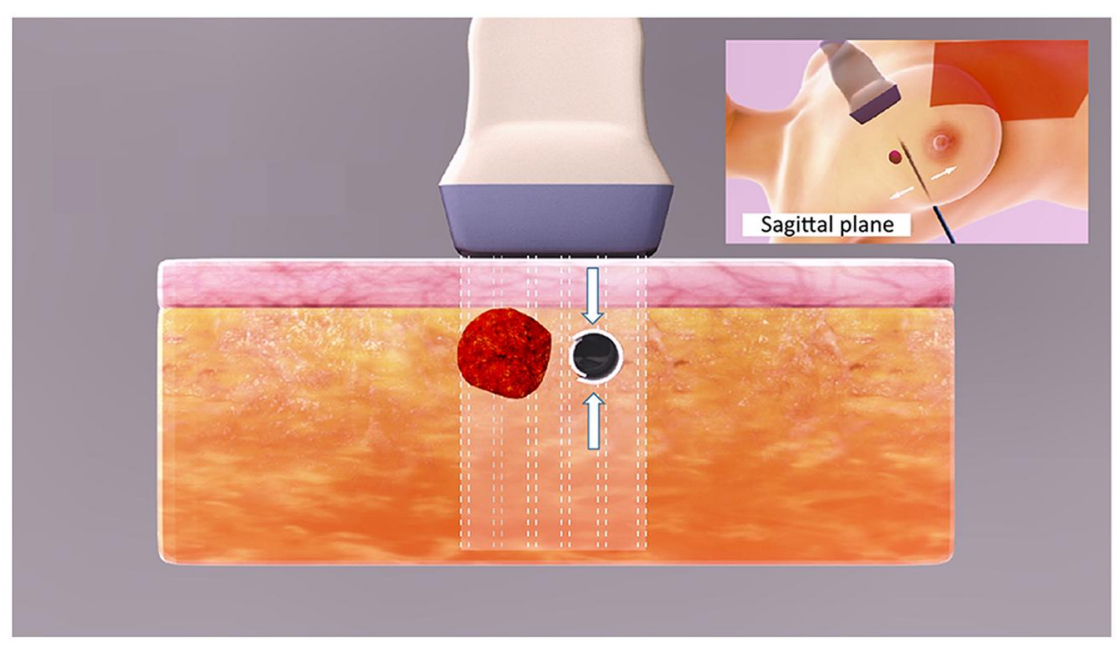

C

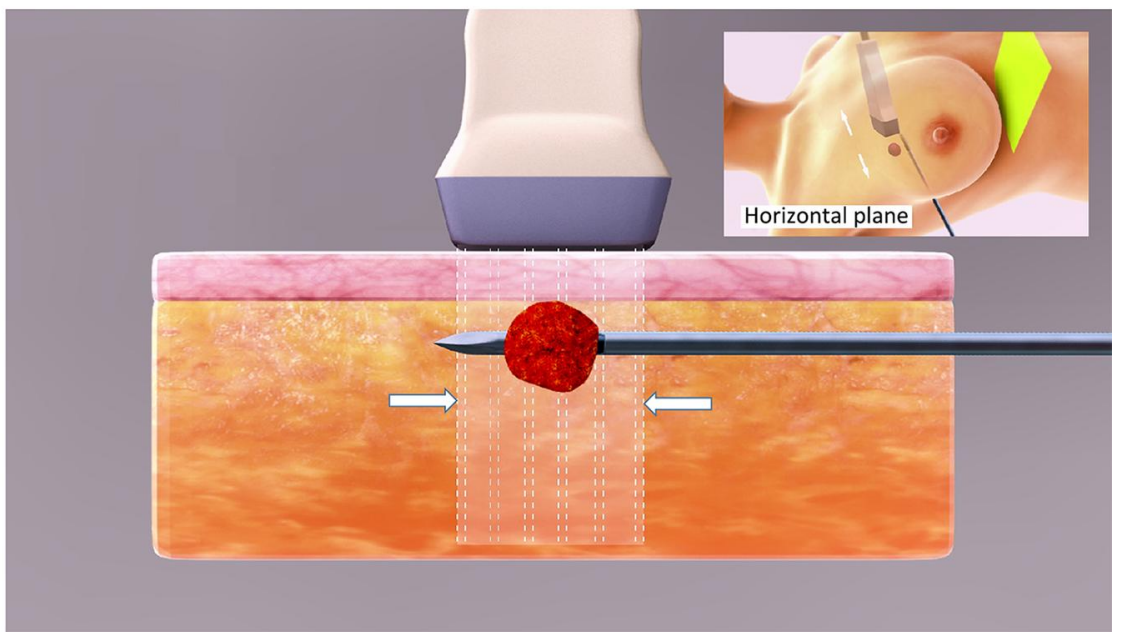

Figure 3 Under the monitoring of ultrasound, through the three-dimensional positioning of the coronal plane, the sagittal plane and the horizontal plane, the rotary cutting knife slot is penetrated to the horizontal positive side of the breast mass, and finally the ultrasound probe is vertical to the rotary cutting knife head, so that the accurate resection of the breast mass under the real-time monitoring of ultrasound is realized. (A) In the first step, the coronal plane (left-right direction) was positioned, and the ultrasonic probe was parallel to the rotary cutter slot. The rotary cutter head was punctured to the side of the breast mass. (B) In the second step, positioning was performed in the sagittal plane (up and down). The ultrasonic probe was perpendicular to the rotary cutter slot, and the ultrasonic probe was punctured to the horizontal side of the breast mass. (C) Thirdly, position in a horizontal plane (frontrear direction), returning that ultrasonic probe to a position parallel to the rotary cut cutter head, and puncturing the rotary cutting cut groove to the horizontal positive side of the breast mass. 
months after the operation, postoperative ecchymosis, and the amount of intraoperative bleeding.

\section{Statistical Treatment}

The Statistical Package for Social Sciences 26.0 was adopted for statistical analysis; the measurement data of normal distribution were expressed as $\bar{x} \pm \mathrm{s}$. The independent sample $t$-test was used to compare the two groups, while the $\mathrm{x}^{2}$ test was used for enumeration data. A p-value of $<0.05$ was considered statistically significant.

\section{Results}

\section{General Information}

This retrospective study included 102 patients with superficial benign breast tumors from July 2017 to June 2019 . There were 69 patients (horizontal group) who underwent the horizontal rotary-cut surgery from July 2018 to June 2019, while 33 patients (traditional group) who underwent the traditional rotary-cut surgery from July 2017 to June 2018. They were aged between 18 and 73 with a mean age of $34.1 \pm 9.4$. Some of the breast masses (36) were found by patients, and 66 breast tumors were discovered by ultrasound in the breast examination, without nipple discharge. They occurred singly. The median diameter of tumors was $1.25 \mathrm{~cm}(0.5-1.9 \mathrm{~cm})$, and the median distance between the upper margin and the skin was $0.6 \mathrm{~cm}(0.4-1.0 \mathrm{~cm})$ under breast ultrasound. Tumor location: 23 tumors near the armpit (lateral margin of the tumor within $2.0 \mathrm{~cm}$ from glandular margins at the armpit), 11 tumors near the sternum (medial margin of the tumor within $2.0 \mathrm{~cm}$ from glandular margins at the sternum), and 68 conventional tumors in other sites (excluding masses within $1.0 \mathrm{~cm}$ around the nipple). For BI-RADS IVA and below, patients over 40 should have a molybdenum target examination to exclude the possible diagnosis of cancer due to calcification. Blood routine, coagulation function, and liver and kidney function were normal. Eight patients had complications with diabetes mellitus (7.8-10.0 mmol/L), with no heart and lung complications, and no history of breast surgery. The difference in comparison of general preoperative data was not statistically significant $(P<0.05)$ but comparable between the two groups in addition to the distance (with statistical differences but no clinical significance) between the upper margin and the skin. See Table 1.

\section{Intraoperative and Postoperative Conditions}

Compared with traditional group, the operation time was significantly shorter and the number of skin damage was significantly less in the horizontal group $(P<0.05)$. Three patients with skin damage were found in the traditional group. The damage was close to the skin with $0.6,0.6$, and $0.7 \mathrm{~cm}$, respectively. The skin margins were sutured and clipped cosmetically. Among the three patients with skin damage, two patients had partial skin damage when less than a third of the tumor was removed. The tumor was $0.6 \mathrm{~cm}$ away from the skin. The skin margin was clipped. The residual part of the tumor was separated and removed under direct vision. Postoperative pathological results in the horizontal group: fibroadenoma $(\mathrm{n}=49)$, adenosis of the breast $(n=12)$, fat calcification $(n=2)$, intraductal papilloma $(\mathrm{n}=3)$, and carcinoma in situ $(\mathrm{n}=2$, one for breast-conserving therapy + sentinel lymph node biopsy and another for mastectomy + sentinel lymph node biopsy due to the failure of breast-conserving because of a long needle passage). Traditional group: fibroadenoma $(n=27)$, adenosis of the breast $(n=4)$, fat calcification $(n=1)$, intraductal papilloma $(n=1)$. For $6-30$ months after the operation, 97 patients were followed up, with a median of

Table I Comparison of General Information Between the Two Groups ( $\bar{x} \pm s)$

\begin{tabular}{|c|c|c|c|c|c|c|c|}
\hline \multirow[t]{2}{*}{ Groups } & \multirow{2}{*}{$\begin{array}{c}\text { Age } \\
\text { (Years) }\end{array}$} & \multirow{2}{*}{$\begin{array}{c}\text { Tumor } \\
\text { Diameter } \\
\text { (cm) }\end{array}$} & \multirow{2}{*}{$\begin{array}{c}\text { Distance Between Upper Edge of } \\
\text { Tumor and Skin }(\mathrm{cm})\end{array}$} & \multicolumn{3}{|c|}{ Tumor Location } & \multirow{2}{*}{$\begin{array}{l}\text { Combinec } \\
\text { Diabetes }\end{array}$} \\
\hline & & & & $\begin{array}{c}\text { Near } \\
\text { Armpit }\end{array}$ & $\begin{array}{c}\text { Near } \\
\text { Sternum }\end{array}$ & $\begin{array}{l}\text { Conventional } \\
\text { Tumors }\end{array}$ & \\
\hline $\begin{array}{l}\text { Horizontal } \\
\text { group }(n=69)\end{array}$ & $34.5 \pm 9.0$ & $1.2 \pm 0.4$ & $0.6 \pm 0.2$ & 16 & 8 & 45 & 5 \\
\hline $\begin{array}{l}\text { Traditional } \\
\text { group }(n=33)\end{array}$ & $33.6 \pm 10.1$ & $1.3 \pm 0.4$ & $0.7 \pm 0.1$ & 7 & 3 & 23 & 3 \\
\hline$t\left(\chi^{2}\right)$ value & $t=0.457$ & $t=-0.64 I$ & $t=-2.455$ & \multicolumn{3}{|c|}{$\chi^{2}=0.236$} & $\chi^{2}=0.105$ \\
\hline$p$-value & 0.648 & 0.523 & 0.016 & \multicolumn{3}{|c|}{0.889} & 0.746 \\
\hline
\end{tabular}


Table 2 Comparison of Intraoperative and Postoperative Conditions Between the Two Groups ( $\bar{x} \pm s)$

\begin{tabular}{|l|c|c|c|c|c|}
\hline Groups & $\begin{array}{c}\text { Surgical Resection } \\
\text { Time }(\mathbf{m i n})\end{array}$ & $\begin{array}{c}\text { Intraoperative } \\
\text { Bleeding }(\mathbf{m L})\end{array}$ & $\begin{array}{c}\text { Skin } \\
\text { Damage }\end{array}$ & $\begin{array}{c}\text { Postoperative } \\
\text { Ecchymosis }\end{array}$ & $\begin{array}{c}\text { Local Residue Six Months After } \\
\text { the Operation }\end{array}$ \\
\hline $\begin{array}{l}\text { Horizontal } \\
\text { group (n=69) }\end{array}$ & $7.7 \pm 1.1$ & $6.0 \pm 1.3$ & 0 & 4 & 0 \\
\hline $\begin{array}{l}\text { Traditional } \\
\text { group (n=33) }\end{array}$ & $9.5 \pm 1.3$ & $6.5 \pm 1.5$ & 3 & 2 & 2 \\
\hline$t\left(\chi^{2}\right)$ value & -7.458 & -1.853 & & 0.000 & \\
\hline$p$-value & 0.000 & 0.067 & $0.032^{*}$ & 1.000 & $0.103^{*}$ \\
\hline
\end{tabular}

Note: *Fisher precision inspection.

$16.5 \pm 4.5$ months. No residue or recurrence was observed in the two groups in the ultrasound review (Table 2).

\section{Discussion}

Originally, the breast rotary-cut technique was used for breast tumor biopsy in the United States. ${ }^{7,8}$ In 2004, the USFDA approved the minimally invasive breast rotary-cut technique for removing benign breast lesions. High-frequency, ultrasound-guided, minimally invasive breast rotary-cut surgery is widely used ${ }^{9}$ to treat benign tumors in clinical practice due to ultrasound convenience and accuracy. At present, it has become the most important minimally invasive surgical method for the removal of benign breast tumors in China. For surgeons, an excision with the rotary cutter groove directly below the breast tumor is considered a classic breast rotary-cut approach, as it is intuitive and easy to understand and perform. Nevertheless, the risk of skin injury in rotary-cut procedures for superficial breast benign tumors is significantly high, especially when the upper margin is located $\leq 1.0 \mathrm{~cm}$ from the skin. During horizontal rotary cutting, the rotary cutter groove is in the true lateral position of the tumor, minimizing skin damage. As the rotary-cut technique is precise under ultrasound, the key issue is achieving accurate positioning.

\section{Three-Step Three-Dimensional Horizontal Rotary-Cut Breast Technique}

In this study, ultrasonic positioning has been divided into three steps, which are more accurate and easier to understand and perform. The first step of the technique is locating the lateral boundary of the breast tumor with the coronal view (ie, left and right direction). The ultrasonic probe is parallel to the rotary cutter groove and moves left and right so that the cutter groove is as close as possible to the lateral side of the breast tumor. The second step is locating the depth of the breast tumor with the sagittal view (ie, up and down directions). The ultrasonic probe is perpendicular to the rotary cutter groove, allowing the rotary cutter groove to be seated on the lateral side of the breast tumor. The third step is locating the relative position of the breast tumor. The rotary cutter groove is in the horizontal view (ie, anterior and posterior directions). The ultrasonic probe returns the rotary cutter groove to the parallel state and moves left and right so that the rotary cutter groove is in the true lateral position of the breast tumor, meaning that the breast tumor is completely within the scope of the rotary cutter groove. Chen et $\mathrm{al}^{10}$ attempted to remove a tumor via a threedimensional, computer-assisted ultrasound. By decomposing three-dimensional positioning into the three above-mentioned steps, we can accurately place the rotary cutter groove into the true lateral position of the breast tumor and then press the cutting key, completing a horizontal, minimally invasive rotary-cut breast tumor surgery.

During horizontal rotary cutting, the ultrasonic probe is perpendicular to the rotary cutter groove. This way, we can observe the transverse section of the breast tumor in order to perform ultrasonic monitoring during rotary cutting. Several scholars also suggest that the transverse section of the tumor should be combined with the resection's longitudinal section during vertical rotary cutting. The operation time and rotary-cut frequencies in the transverse section method are lower than in the longitudinal section method. Furthermore, the tumor recurrence rate is reduced in the transverse section method compared with the longitudinal section method. ${ }^{11}$ However, few reports have been made on rotary lateral tumor cutter grooves. Ultrasonic probe location methods are also rarely discussed in detail.

\section{Horizontal Rotary-Cut for Skin Damage and Tumor Residue Reduction}

Horizontal rotary-cut advantages: first, for superficial breast tumors, the suction effect of the rotary cutter bit 
on the subcutaneous tissue above the tumor is minimized, thus avoiding skin damage above the superficial tumor. Second, the ultrasonic probe is perpendicular to the cutter groove and short tumor diameter. This can help achieve complete ultrasonic monitoring for breast tumor removal, higher surgery accuracy, and a lower residual rate. The residual rate is also closely related to tumor size. ${ }^{12}$ The total resection rate can reach up to $98 \% .^{13}$ Rotary-cut therapy ${ }^{14}$ is not recommended for breast tumors with a diameter over $3.0 \mathrm{~cm}$.

Compared with skin damage, bleeding is not the main challenge to be overcome. The thickness of skin and dermis is generally $0.05-0.4 \mathrm{~cm} .{ }^{15}$ Therefore, the minimum distance between the upper edge of the tumor and the skin in this study has been defined as $0.4 \mathrm{~cm}$. Patients in the traditional group received traditional vertical rotary-cut therapy. There were 33 cases of removed superficial breast mass in this group. Because of the risk of skin damage on the surface of the tumor due to the rotary cutter groove inhaling it, an assistant was required to help stretch the skin and administer subcutaneous injections of a local anesthetic to form an isolation zone. Therefore, the operation was relatively prolonged. Despite this, in the control group, 3 patients had skin damage and 2 patients had partial residues due to concerns during the operation in cases where the upper margin of the tumor was close to the skin. The residual part of the tumor had to be removed under open and direct vision through the damaged skin. The skin edge was then clipped and cosmetically sutured. Patients in the horizontal group received horizontal rotary-cut therapy to remove 69 superficial breast tumors. Concerns about skin damage were significantly reduced; thus, even though the distance between the upper edge of the tumor and the skin was smaller (minimum distance of $0.4 \mathrm{~cm}$ vs $0.6 \mathrm{~cm}$ ), the average operation time was significantly shorter ( 7.7 minutes vs 9.5 minutes) than in the traditional group. Moreover, no skin damage and tumor residues were present.

Because Asian women's breasts are relatively small, their mammary glands and subcutaneous fat layers are noticeably thinner than in women of other ethnicities. Thus, horizontal rotary-cut therapy is more suitable for Asian women than traditional vertical rotary-cut therapy.

\section{Adjustment of Patient Positions for Cutter Groove or Tumor Displacement Avoidance}

During breast rotary-cut surgery, ultrasonic positioning must be accurate. This includes initial positioning and positioning during rotary cutting. In breast rotary-cut surgery, the threestep three-dimensional positioning method may achieve rapid and accurate initial breast tumor positioning. However, the key to achieving accurate positioning during rotary cutting is avoiding the displacement of the rotary cutter groove or the tumor. In a side-puncture mouth, the rotary cutter groove is generally easy to shift or tilt upward, while in an areolapuncture mouth, the rotary-cutter groove is generally inclined toward a downward shift. Breast motion is increased in large breasts, making it easy to shift the breast tumor lower. In the three above-mentioned cases, adjusting the patient's position places the breast tumor at the top of the chest wall, allowing the handle of the rotary-cutter groove to work horizontally. This reduces inaccuracy caused by the displacement of the rotary cutter bit or the tumor during the operation, thus reducing the operation's difficulty and increasing its success rate.

\section{Bleeding in Superficial Breast Tumor Rotary-Cut Surgery}

Intraoperative hemorrhage, hematoma formation, and skin ecchymosis are the most common complications of rotarycut surgery. Although color ultrasound can detect blood vessels around and away from the tumor during surgery, the bleeding-related complication rate remains $2.54-5.52 \%{ }^{16}$ In superficial breast masses, it is also common to injure the subcutaneous capillary network and cause bleeding during rotary cutting. However, because the tumor is superficial and close to the residual cavity after the rotary cutting, local compression can be applied easily to stop the bleeding quickly and effectively. Therefore, the bleeding-related complication rate of superficial masses is relatively low, and the complications are usually skin congestions, rather than hematomas.

In regard to the treatment of intraoperative bleeding, our experience concludes as follows. Once there is a small amount of bleeding during the operation, the focus should be on quick, accurate, and complete lesion removal and stopping the bleeding with precise compression. On the one hand, this procedure ensures precise removal of the lesion and avoids residue due to bleeding, hemostasis, and final tumor resection. Thus, it can save operation time and help avoid massive bleeding. In the case of uncontrollable active bleeding, a small incision should be made on the surface of the tumor, and an electric knife should be used to stop the bleeding and avoid serious adverse consequences. 
This study does not include multiple tumor surgery. For multiple masses, our principle is to remove one mass and compress the residual cavity to ensure that the next mass is removed when there is no active bleeding. However, due to the change of the location of the tumor and the adjustment of its position, continual pressing can no longer be used to stop bleeding in the residual cavity of the first removed tumor in order to remove the next tumor. Consequently, the incidence of skin ecchymosis after rotary cutting of multiple tumors is higher than after rotary cutting of a single tumor.

\section{Preoperative, Intraoperative, and Postoperative Reviews After Mammary Gland Scale Membrane Application}

The practical problem at hand is ensuring that rotary-cut breast surgery is accurate and simple in terms of preoperative body surface localization, intraoperative reduction, and postoperative review. The body surface positioning scale membrane for breast tumors is a patented technical product (patent no.: ZL 201020172673.2) of our department. It is used in breast rotary-cut surgery for preoperative localization and intraoperative reduction as well as postoperative review comparisons before and after operations in tumors requiring removal. This increases the patient's chances of surgical safety and helps avoid unnecessary medical disputes. Preoperative positioning is usually completed immediately after the surgeon establishes the procedure in combination with the ultrasound report. Intraoperative reduction may save secondary positioning time. It is necessary to use the new mammary gland scale membrane to locate the newly discovered tumor in the postoperative ultrasound review and compare it with the preoperative marked mammary gland scale membrane in order to determine whether there is any residue or recurrence in the resection of the tumor. In this study, the mammary gland scale membrane was used routinely in both the horizontal group and the traditional group. Therefore, accurate two-dimensional clock positioning is guaranteed before, during, and after surgery. Moreover, the ultrasonic examination in the same position determines whether there is any residue or recurrence.

\section{Inclusion Criteria for Breast Tumors with BI-RADS IVA Under Ultrasound}

Breast rotary-cut surgery for breast tumors with BI-RADS IVA under ultrasound has been approved ${ }^{17}$ by several specialists; however, it is still controversial. ${ }^{18}$ BI-RADS IVA suggests a $5-10 \%$ possibility of malignancy. The rotary-cut surgery for these patients may enable over $90 \%$ of patients to receive minimally invasive rotary-cut surgery. However, because breast rotary-cut surgery generally chooses the areola or the lateral margin of the breast for the needle entry, the angle between the needle and the chest wall is generally less than $30^{\circ}$ in order to avoid pneumothorax, resulting in a relatively long needle channel. For this reason, a number of scholars believe that several patients with breast cancer will lose the chance for breast-conserving surgery after the partial rotary-cut surgery. Ohsumi et $\mathrm{al}^{19}$ found that there is no significant increase in the local recurrence rate after breast-conserving surgery, although needle passage metastasis after breast rotary-cut surgery is present. However, the consensus at home and abroad is that breast rotary-cut is only recommended for the treatment of benign breast tumors and suspected malignant lesions under biopsy. It has not yet been applied in radical mastectomy ${ }^{20}$ for early breast cancer. Therefore, secondary surgery is inevitable in patients with breast cancer confirmed by pathology after breast rotary-cut surgery.

\section{Conclusions}

In superficial benign breast tumors with $\leq 1.0 \mathrm{~cm}$ between the upper margin of the tumor and the skin, the horizontal rotary-cut breast technique may help avoid skin damage on the surface of the tumor, shorten the operation time, reduce the tumor residue, and complete the whole procedure visualization under ultrasound. The three-step threedimensional positioning standardizes and simplifies the process of breast tumor positioning under ultrasound and reduces the difficulty of accurate positioning. It is also easy to learn and operate. The three-step threedimensional positioning horizontal rotary-cut breast technique has both a popularization and application value.

\section{Acknowledgments}

We are particularly grateful to all the people who have given us help on our article.

\section{Funding}

This study was funded by the Liaoning Province Appropriate Technology Promotion Project for Grassroots Health (2017-284-15) and the second batch of flexible introduction of leading talents in Xining City under the "555 Plan for Attracting Talents and Gathering 
Talents" project (2019-4). The funding body had no role in the design of the study and collection, analysis, and interpretation of data and in writing the manuscript.

\section{Disclosure}

The authors declare that they have no competing interests.

\section{References}

1. Zannis VJ, Aliano KM. The evolving practice pattern of the breast surgeon with disappearance of open biopsy for nonpalpable lesions. Am J Surg. 1998;176(6):525-528. doi:10.1016/S0002-9610(98)002657

2. Kibil W, Hodorowicz-Zaniewska D, Kulig J. Mammotome biopsy under ultrasound control in the diagnostics and treatment of nodula breast lesions - own experience. Pol Przegl Chir. 2012;84(5):242-246. doi:10.2478/v10035-012-0040-1

3. Fan ZM, Wang JD, Hua B, et al. Expert consensus and operational guidelines for ultrasound-guided vacuum-assisted breast biopsy procedures (2017 edition). Chin J Pract Surg. 2017;37(12):1374-1376. [Article in Chinese].

4. Li RX, Guo JJ, Zhu ZL. Application of local tumescent anesthesia technique in minimally invasive Mammotome rotation cutting for multiple solid breast lesions. Chin J Gen Surg. 2017;26(05):619-625. [Article in Chinese].

5. Song RF, Cui JC, Yu XW, et al. Feasibility study of horizontal mastectomy for superficial benign breast masses. Chin J Minim Invasive Surg. 2020;20(12):1106-1110.

6. Cui JC. Three dimensional horizontal minimally invasive surgery for breast tumor: three steps [J/CD]. Chin Arch Gen Surg. 2021;15(1):74.

7. Sohn YM, Yoon JH, Kim EK, Moon HJ, Kim MJ. Percutaneous ultrasound-guided vacuum-assisted removal versus surgery for breast lesions showing imaging-histology discordance after ultrasound-guided core-needle biopsy. Korean J Radiol. 2014;15 (6):697-703. doi:10.3348/kjr.2014.15.6.697

8. Nakano S, Otsuka M, Mibu A, Oinuma T. Significance of fine needle aspiration cytology and vacuum-assisted core needle biopsy for small breast lesions. Clin Breast Cancer. 2015;15(1):e23-e26. doi:10.1016/j. clbc.2014.07.001

9. Yamaguchi R, Tanaka M, Tse GM, et al. Management of breast papillary lesions diagnosed in ultrasound-guided vacuum-assisted and core needle biopsies. Histopathology. 2015;66(4):565-576. doi:10.1111/his.12477
10. Chen DR, Chang RF, Chen CJ, Chang CC, Jeng LB. Threedimensional ultrasound in margin evaluation for breast tumor excision using Mammotome. Ultrasound Med Biol. 2004;30(2):169-179. doi:10.1016/j.ultrasmedbio.2003.10.004

11. Shi YY, Li HN. Effect of ultrasound-guided minimally invasive circumflex mastectomy on longitudinal and transverse sections. Med Innov China. 2019;16(28):127-130. [Article in Chinese].

12. Papathemelis T, Heim S, Lux MP, Erhardt I, Scharl A, Scharl S. Minimally invasive breast fibroadenoma excision using an ultrasound-guided vacuum-assisted biopsy device. Geburtshilfe Frauenheilkd. 2017;77(2):176-181. doi:10.1055/s-0043-100387

13. Bromberg SE, Moraes PRAF, Ades F. Prime incision: a minimally invasive approach to breast cancer surgical treatment-A 2 cohort retrospective comparison with conventional breast conserving surgery. PLoS One. 2018;13(1):e0191056. doi:10.1371/journal. pone. 0191056

14. Li S, Wu J, Chen K, et al. Clinical outcomes of 1578 Chinese patients with breast benign diseases after ultrasound-guided vacuum-assisted excision: recurrence and the risk factors. Am J Surg. 2013;205 (1):39-44. doi:10.1016/j.amjsurg.2012.02.021

15. Li YN, Li ZX, Lu YH, et al. High-frequency ultrasound measurement and sonographic study of skin thickness in normal adults. Chin J Med Imaging Technol. 2008;10:1622-1624. [Article in Chinese].

16. Ding B, Chen D, Li X, Zhang H, Zhao Y. Meta analysis of efficacy and safety between Mammotome vacuum-assisted breast biopsy and open excision for benign breast tumor. Gland Surg. 2013;2(2):69-79. doi:10.3978/j.issn.2227-684X.2013.05.06

17. Zhou YG, Duan YY, Zhao HD, et al. Application of preoperative ultrasound evaluation and precise positioning in treatment of breast multiple benign masses by vacuum assisted minimally invasive biopsy system. J Clin Ultrasound Med. 2019;21(03):212-214. [Article in Chinese].

18. Lakoma A, Kim ES. Minimally invasive surgical management of benign breast lesions. Gland Surg. 2014;3(2):142-148. doi:10.3978/ j.issn.2227-684X.2014.04.01

19. Ohsumi S, Taira N, Takabatake D, et al. Breast biopsy for mammographically detected nonpalpable lesions using a vacuum-assisted biopsy device (Mammotome) and upright-type stereotactic mammography unit without a digital imaging system: experience of 500 biopsies. Breast Cancer. 2014;21(2):123-127. doi:10.1007/s12282012-0360-3

20. Bennett IC, Saboo A. The evolving role of vacuum assisted biopsy of the breast: a progression from fine-needle aspiration biopsy. World J Surg. 2019;43(4):1054-1061. doi:10.1007/s00268-018-04892-x
International Journal of General Medicine

\section{Publish your work in this journal}

The International Journal of General Medicine is an international, peer-reviewed open-access journal that focuses on general and internal medicine, pathogenesis, epidemiology, diagnosis, monitoring and treatment protocols. The journal is characterized by the rapid reporting of reviews, original research and clinical studies across all disease areas. The manuscript management system is completely online and includes a very quick and fair peer-review system, which is all easy to use. Visit http://www.dovepress.com/ testimonials.php to read real quotes from published authors. 